\title{
A study of Neonatal Sepsis and to assess the validity of sepsis screen as a diagnostic tool
}

\author{
Juneja S. ${ }^{1}$, Chaudhary P. ${ }^{2}$, Thora $^{3}$, Arya S. ${ }^{4}$ \\ ${ }^{1}$ Dr. Sunny Juneja, PG student, ${ }^{2}$ Dr. Prachi Chaudhary, Assistant Professor, ${ }^{3}$ Dr. S Thora, Professor and HOD, ${ }^{4}$ Dr. Sunil \\ Arya, Assistant Professor, all authors are affiliated with Department of Pediatrics, MGM Medical College and Associated \\ MY Hospital Indore.
}

Corresponding Author: Dr. Prachi Chaudhary, A -504, Sky Heights Apartment, Road, Near Navlakha Square, Indore.

\begin{abstract}
Introduction: Neonatal sepsis is the leading cause of neonatal mortality in the developing world. There is an increasing incidence of drug resistance among organisms. We have tried to the sepsis profile in our nursery and to assess the validity of sepsis screen as a diagnostic tool. We have also studied the individual parameters of the screen for their strength as a diagnostic tool. Methods: This was a prospective study carried out in the SCNU of MY Hospital and included 200 newborns with suspected sepsis. Sepsis Screen and Blood Cultures were done in all the babies and the results were analysed. Results: Out of the 200 patients with suspected sepsis, 150 (75\%) had a positive sepsis screen whereas 102 (51\%) were culture positive. Sepsis Screen was positive in $93.1 \%$ of total culture positive sepsis and in $56.1 \%$ of culture negative cases too. Thus, the Sensitivity and specificity for Septic Screen was calculated to be 93.18 and $43.88 \%$ repetitively. PPV was $63.33 \%$ and NPV was $86.0 \%$. Conclusion: The bacteriological flora has changed over the last 12 years and there has been a high incidence of resistance to the first live antibiotics in our Nursery. The sepsis screen has a high negative predictive value and it is recommended that negative sepsis screen should warrant discontinuation of the empirical antibiotics to prevent the unnecessary use and the emergence of drug resistant organisms.
\end{abstract}

Key words: Culture positive sepsis, Neonatal sepsis, Sepsis Screen

\section{Introduction}

Neonatal sepsis is the leading cause of neonatal mortality. It is responsible for $13 \%$ of all neonatal mortality, and $42 \%$ of deaths in the first week of life $[1,2]$. The multiple skin punctures and invasive procedures that preterm newborns commonly undergo increase even more the risk of infections in this population.

In developing countries, clinically diagnosed sepsis is present in 49-170 per 1000 live births, culture-proven sepsis in 16 per 1000 live births and neonatal meningitis in $0.8-6.1$ per 1000 live births[3].

Even though a positive blood culture, is gold standard for diagnosis of neonatal sepsis the technique is time consuming, demands a proper laboratory setup and is positive in only $40 \%$ cases. Early treatment with antibiotics is possible with the help of certain indirect

Manuscript received: $4^{\text {th }}$ February 2018

Reviewed: $14^{\text {th }}$ February 2018

Author Corrected: $20^{\text {th }}$ February2018

Accepted for Publication: $26^{\text {th }}$ February 2018 markers such as neutropenia $\left(<1800\right.$ cells $\left./ \mathrm{mm}^{3}\right)$, leucopenia $\left(<5000\right.$ cells $\left./ \mathrm{mm}^{3}\right)$, band cells, I/T ratio of $>$ 0.2 , Platelet Count of $<150000 /$ cumm micro ESR $>15 \mathrm{~mm}$ in 1 st hour and C-reactive protein (CRP) value of $>1 \mathrm{mg} / \mathrm{L} \quad[4,5]$.All these investigations are collectively known as sepsis screen and aids in early diagnosis of neonatal sepsis in absence of negative blood cultures. This screen has been used for a long time for the diagnosis of suspected and probable sepsis. We tried to calculate the sensitivity, specificity positive and negative predictive value of the sepsis screen to diagnose the proven sepsis. We also tried to assess the strength of individual parameters as a diagnostic tool for sepsis.

\section{Materials and Methods}

Place and type of study: The prospective study was carried out in SNCU (Special Care Newborn Unit) of M.Y.Hospital, Department of Pediatrics, MGM Medical College, Indore (M.P.) 
Sampling: A total of 200 newborns, inborn and out born admitted in SNCU of M.Y. hospital, Indore were taken up for study over a period of 6 months in 2016 from May to October.

Inclusion criteria:

Neonates were enrolled based on signs and symptoms of clinical sepsis [as per NNF (National Neonatology Forum) criteria] $[5,6]$. The clinical criteria considered (NNF criteria) were-poor feeding, irritability/ excessive cry, lethargy poor cry and reflexes, fever, hypothermia, jaundice, vomiting, abdominal distension, tachypnoea and grunting, convulsions, diarrhea, pustules, sclerema, cyanosis, bulged fontanelle, DIC/ bleeding, poor perfusion / shock, Apnea. Also, significant predisposing factors for presumed early onset sepsis was taken into consideration (according to NNF guidelines) during inclusion of cases [5,7].

Exclusion criteria: Major congenital anomalies like tracheoesophageal fistula, malrotation of the gut, lobar agenesis of lungs, congenital heart disease or anomalies of the CNS were excluded from the study.
Methods: Following investigations were done in all the cases,Hb, T \& D, Band Cell Count, Thrombocyte count, Immature /Total neutrophil ratio, CRP, micro ESR and blood culture.

The sepsis screen comprised of Total leukocyte count $<$ 5000 cells $/ \mathrm{mm} 3$, I/T ratio $>0.2$, ANC (Absolute Neutrophil Count $)<1800$ cells/mm3, micro-ESR $>15$ $\mathrm{mm}$ at the end of $1 \mathrm{st}$ hour, $\mathrm{C}$ reactive protein $>1 \mathrm{mg} / \mathrm{dl}$.

If any two of the following parameters are positive or significant, the sepsis screen is said to be positive as per NNF guidelines

All the study parameters were entered in the excel sheet and were analysed using epi-info software. Descriptive parameters were used for the univariate analysis.

Sensitivity, specificity, Negative Predictive Value (NPV) and Positive Predictive Value (PPV) of septic screen was compared with culture outcome (gold standard) using a contingency table.

\section{Results}

The general characteristics of study population are shown in table 1 . Total 200 newborns were included in the study out of which $64.5 \%$ were outborns and $35.5 \%$ were inborns. $47 \%$ had early onset sepsis, while $53 \%$ had late onset sepsis. There were $62 \%$ male and $38 \%$ female.

The ratio of male to female is nearly 1.6:1. 8.5\% were less than 32 weeks gestation, $45 \%$ between 32 to 37 weeks and $46.5 \%$ more than 37 weeks. $33.5 \%$ were VLBW, $41.5 \%$ LBW and $25 \%$ had a birth weight of more than $2.5 \mathrm{~kg}$.

Table-1: General study population characteristics

\begin{tabular}{|c|c|c|}
\hline \multirow{2}{*}{ Gender } & Males & $62 \%$ \\
\cline { 2 - 3 } & Females & $38 \%$ \\
\hline \multirow{2}{*}{ Gestational age } & $<32$ weeks & $8.5 \%$ \\
\cline { 2 - 3 } & $32-37$ weeks & $45 \%$ \\
\cline { 2 - 3 } & $>37$ weeks & $46.5 \%$ \\
\hline \multirow{2}{*}{ Place of birth } & Inborn & $35.5 \%$ \\
\cline { 2 - 3 } & Outborn & $64.5 \%$ \\
\hline \multirow{2}{*}{ Birth weight } & $<1.5 \mathrm{~kg}$ & $33.5 \%$ \\
\cline { 2 - 3 } & 1.5 to $2.5 \mathrm{~kg}$ & $41.5 \%$ \\
\cline { 2 - 3 } & $>2.5 \mathrm{~kg}$ & $25 \%$ \\
\cline { 2 - 3 } & EOS & $47 \%$ \\
\hline \multirow{2}{*}{ Onset of sepsis } & LOS & $53 \%$ \\
\cline { 2 - 3 } & &
\end{tabular}

The newborns were included if they had the signs and symptoms of probable sepsis according to the NNF criteria. Table 2 shows the distribution of various major presenting complaint. Respiratory distress was the most common major presentation found in $56 \%$ of patients. 
Table-2: Distribution of cases according to clinical presentation

\begin{tabular}{|c|c|c|}
\hline & Number & Percent \\
\hline Abdominal Distention & 2 & 1 \\
\hline Aponea & 8 & 19.0 \\
\hline Dullness & 39 & 1.5 \\
\hline Hypothermia & 3 & 5.0 \\
\hline Jaundice & 10 & 56.0 \\
\hline Respiratory distress & 112 & 10.5 \\
\hline Refusal to feed & 21 & 2.5 \\
\hline Seizures & 5 & $\mathbf{1 0 0 . 0}$ \\
\hline Total & $\mathbf{2 0 0}$ & \\
\hline
\end{tabular}

Out of the 200 patients with suspected sepsis, 150 (75\%) had a positive sepsis screen whereas $102(51 \%)$ were culture positive. Table 2 The most common organism isolated was Klebsiella pneumoniae in $18.5 \%$ cases followed by $15.5 \%$ of staphylococcus aureus \& $8.5 \%$ of E-Coli, $4.5 \%$ of Enterococcus, $3.5 \%$ of Pseudomonas, \& $0.5 \%$ of Citrobacter.

Table- 3: Correlation between culture positive sepsis and sepsis screen result.

\begin{tabular}{|c|c|c|c|c|}
\hline \multirow{2}{*}{ Septic Screen } & Culture negative & Culturepositive & Total \\
\hline \multirow{2}{*}{ Negative } & Number & 43 & 7 & 50 \\
\cline { 2 - 5 } & Percentage & 43.9 & 6.9 & 25 \\
\hline \multirow{2}{*}{ Positive } & Number & 55 & 95 & 150 \\
\cline { 2 - 5 } & Percentage & 56.1 & 93.1 & 75 \\
\hline \multirow{2}{*}{ Total } & Number & 98 & 102 & 200 \\
\cline { 2 - 5 } & Percentage & $100.0 \%$ & $100.0 \%$ & $100.0 \%$ \\
\hline
\end{tabular}

The above table shows that Sepsis Screen was positive in $93.1 \%$ of total culture positive sepsis and negative in $6.9 \%$. It was positive in $56.1 \%$ of culture negative cases too. Thus, the Sensitivity and specificity for Septic Screen was calculated to be 93.18 and $43.88 \%$ repetitively. PPV was $63.33 \%$ and NPV was $86.0 \%$.

The individual parameters of the sepsis screen were also assessed for their strength of prediction of sepsis. Table 4 shows the values of specificity, sensitivity, PPV and NPV for the different parameters.

Table 4: Table showing values of specificity, sensitivity, PPV and NPV for the different parameters.

\begin{tabular}{|c|c|c|c|c|}
\hline Parameter & Sensitivity & Specificity & PPV & NPV \\
\hline Micro-ESR & 48.02 & 73.47 & 65.33 & 57.60 \\
\hline CRP & 87.25 & 45.92 & 62.68 & 77.59 \\
\hline I/T ratio & 80.39 & 49.98 & 62.12 & 70.59 \\
\hline Platelet count & 91.18 & 35.71 & 59.62 & 79.55 \\
\hline Leukopenia & 52.54 & 52.26 & 64.58 & 42.86 \\
\hline
\end{tabular}

Positive M-ESR was seen in $48 \%$ of total sepsis cases in positive culture and $26.5 \%$ in culture negative cases including both inborn and outborn. Sensitivity and specificity for M-ESR was 48.04 and $73.47 \%$ repetitively. PPV was $65.33 \%$ and NPV was $57.60 \%$

Positive CRP was seen in $87.3 \%$ of total sepsis cases in positive culture and $54.1 \%$ in culture negative cases including both inborn and outborn. Sensitivity and specificity for CRP was 87.25 and $45.92 \%$ repetitively. PPV was $62.68 \%$ and NPV was $77.59 \%$ 
Positive I/T ratio was seen in $57.8 \%$ of total sepsis cases in positive culture and $58.2 \%$ in culture negative cases including both inborn and outborn. Sensitivity and specificity for I/T ratio was 80.39 and $49.98 \%$ repetitively. PPV was $62.2 \%$ and NPV was $70.59 \%$

Thrombocytopenia was seen in $91.2 \%$ of total sepsis cases in positive culture and $87.8 \%$ in culture negative cases including both inborn and outborn. Sensitivity and specificity for Thrombocytopenia was 91.18 and $35.71 \%$ repetitively. PPV was $59.62 \%$ and NPV was $79.55 \%$

Leucopenia was seen in $56.9 \%$ of total sepsis cases in positive culture and $65.3 \%$ in culture negative cases including both inborn and out born. Sensitivity and specificity for Leukopenia was 52.54 and $52.26 \%$ repetitively. PPV was $64.58 \%$ and NPV was $42.86 \%$

\section{Discussion}

Blood culture has remained the gold standard for the confirmation of sepsis. In our study, $51 \%$ neonates with suspected sepsis had positive cultures. Other authors have observed culture positivity in 30 to $55 \%$ patients in different studies [810]. At advanced centres, blood culture is positive in upto $80 \%$ of genuine sepsis [11]. Thus culture positivity rate is highly variable from place to place.

The most common organism isolated in present study was Klebsiella pneumoniae followed by staphylococcus aureusfollowed by E.Coli. This finding is in accordance with NNPD 2002-03 data, where the most common organisms causing neonatal sepsis was Klebsiella pneumoniae followed by staphylococcus aureus and pseudomonas [12]. However other studies have reported Staphylococcus aureus as the commonest organism to be isolated [13].

In our study, when comparing early onset and late onset sepsis, we found that Klebsiella pneumoniae was the most common isolate in early onset sepsis while in late onset sepsis it was Staphylococcus aureus. While in the developed world Group B Streptococcus is the commonest organism responsible for Early onset sepsis which is quite in contrast to the developing world [14].

In our study, both Gram-positive and Gram-negative isolates showed a high resistance to cephalosporins, penicillin, gentamycin and amoxiclav. Thakur et al observed that antibiotic resistance among the Gram-positive isolates was highest to penicillin (87\%) followed by amoxyclav (66\%)[15]. Reports of high resistance to Ampicillin (71\%) has also been reported by Bhat et al [16]. In the current study most of the Gram-positive isolates were sensitive to vancomycin which is also seen in the study by Hoogen et al [17].

Gram-negative isolates showed a high resistance to all cephalosporins which is like the resistance pattern reported by Agnihotri et al[18] and Bhat et al[16,18].This high resistance pattern could be attributed to the injudicious use of antibiotics in our region.

In a study conducted by Dr Zafar Khan 2003 in our NICU, it was found that E Coli was the commonest organism isolated in newborns with sepsis followed by Klebsiella pneumoniae. That time the isolated E coli was mostly susceptible to ciprofloxacin and Klebsiella isolates were sensitive to amikacin.

But in the present scenario both the organisms are resistant to quinolones as well as aminoglycosides and only sensitive to meropenem and colistin. This shows that bacteriological profile and the sensitivity pattern has changed over a period and the organisms have gained resistance to the first and second line therapy.

In the present study, overall mortality was observed in $29 \%$, whereas Chaudhary reported a mortality of $45.5 \%$ in their study, which is quite high as compared to our study. Thakur et al also reported the low mortality rate (11.7\%). This could be attributed to advancement in medical technology and better neonatal care in NICU.

Two or more abnormal parameters of the sepsis screen had a high accuracy in predicting neonatal sepsis. While comparing the validity of sepsis screen results between various studies, a lot of variation has been noticed. The table below compares the values among various studies. 
Original Research Article

Table 5: Comparison of sepsis screen validity in different studies.

\begin{tabular}{|c|c|c|c|c|c|c|}
\hline No. & Authors & Year & Sensitivity (\%) & $\begin{array}{c}\text { Specificity } \\
\mathbf{( \% )}\end{array}$ & $\begin{array}{c}\text { Positive predictive } \\
\text { value (\%) }\end{array}$ & $\begin{array}{c}\text { Negative predictive } \\
\text { value (\%) }\end{array}$ \\
\hline 1 & Gerdes et al. & 2004 & 100.0 & 83.0 & 27.00 & 100.0 \\
\hline 2 & Sriram et al. & 2011 & 55.30 & 91.70 & 98.30 & 19.30 \\
\hline 3 & $\begin{array}{c}\text { Swarnakar et } \\
\text { al. }\end{array}$ & 2012 & 56.0 & 87.50 & 97.00 & 20.00 \\
\hline 4 & Jadhav et al. & 2013 & 100.0 & 62.50 & 63.30 & 100.0 \\
\hline 5 & Vinay et al, & 2015 & 77 & 41 & 84 & 31 \\
\hline 6 & Bhale et al. & 2015 & 93.4 & 77.0 & 78.7 & 92.77 \\
\hline 7. & $\begin{array}{c}\text { Present } \\
\text { Study }\end{array}$ & 2016 & 93.18 & 43.88 & 63.33 & 86.0 \\
\hline
\end{tabular}

Some studies have reports very high sensitivities and negative predictive value up to $100 \%$ while some reported very high specificities and positive predive value upto 92 to $97 \%$ [19-24]. Our study found a high sensitivity and NPV of the sepsis screen of $93.18 \%$ and $86 \%$ respectively whereas slightly lower specificity and PPV of $43.88 \%$ and $63.33 \%$ respectively. The sensitivity results in the present study were in accordance with Gerdes et al, Jadhav et al., and Bhale et al $[19,22,24]$.

When comparing the individual parameters of sepsis screen, platelet count was found to be the most sensitive indicator of sepsis followed by CRP and I/T ratio in order. And micro ESR was found to be the most specific indicator of sepsis. Thesensitivity, Specificity, PPV and NPV of the platelet count as an individual parameter were very comparable to the full sepsis screen.

\section{Conclusion}

The organisms causing sepsis have changed over time, with Klebsiella now being the most common as compared to E.Coli earlier (2003 study). Also, there is a marked prevalence of antibiotic resistance among the prevalent organisms.

The sepsis screen is quick and is helpful in differentiating possible sepsis from probable sepsis. As sepsis screen has a high Negative Predictive Value, its main value remains in excluding the infections rather than confirming sepsis. If the sepsis screen is negative in the presence of strong clinical suspicion, it should be repeated within 12 hours. If the screen is still negative, sepsis can be excluded with reasonable certainty. Excluding sepsis with sepsis screen will make possible more rationale use of antibiotics and limit the empirical use. This will prevent development of resistance as well as save money spent on neonatal care.

We studied the individual parameter of septic screen and found that platelet count is the most sensitive predictor of sepsis followed by CRP. Thus, if a neonate has a normal platelet count and CRP, sepsis can be quite reasonably excluded. Normal platelet count and normal CRP can reasonably assure us to withhold antibiotics. Limiting antibiotics overuse is of utmost importance in a NICU.
As platelet count has near comparable Sensitivity, Specificity, PPV and NPV as the full sepsis screen, other studies which may include a higher number of newborns may be conducted to see whether thrombocytopenia alone along with the clinical signs of sepsis is good enough to call it as probable sepsis instead of the full screen. This might be useful in resource poor settings.

We also recommend yearly or time to time analysis of sepsis in all nurseries with their bacteriological profile and their sensitivity pattern, which will help to prevent antibiotic resistance.

\section{What this study adds?}

1. The NPV and sensitivity of sepsis screen is very high so its main use should in excluding infection rather than confirming it.

2. Drug resistant strains of Klebsiella pneumoniae is the most common organism in present situation.

3. Normal platelet count can be very helpful and reassuring especially in resource poor settings to exclude sepsis.

Source of funding: none, in house investigatory facilities were used and outsourcing was utilized as per the institutional norms. 
Contribution details: Dr Sharad Thora and Dr Prachi Chaudhary had planned, designed, guided the study and analysed the data. Dr Sunny Juneja had collected the data and analysed it. Dr Sunil Arya analysed the data and helped in preparing the manuscript.

Funding: Nil, Conflict of interest: None initiated, Perission from IRB: Yes

\section{References}

1. Lawn JE, Cousens S, Zupan J, et al. 4 million neonatal deaths: when? Where? Why? Lancet 2005; 365: 891-900

2. Liu L, Johnson HL, Cousens S, et al. Global, regional, and national causes of child mortality: an updated systematic analysis for 2010 with time trends since 2000. Lancet 2012; 379:2151-61

3. Thaver D, Zaidi AKM. Burden of neonatal infections in developing countries: a review of evidence from community based studies. Pediatr Infect Dis J 2009; 28 (1 Suppl): S3-9.

4. Gerdes JS, Polin R. Early diagnosis and treatment of neonatal sepsis. Indian J Pediatr; Jan-Feb 65(1): 63-78.

5. Da Silva O, Ohlsson A, Kenyon C. Accuracy of leukocyte indices and C-reactive protein for diagnosis of neonatal sepsis: a critical review. Pediatr Infect Dis J 1995; 14:362-6

6. WHO Handbook IMCI Integrated Management of Childhood Illnesses. WHO/ FCH/ CAH/00. 12 Geneva: WHO 2000.

7. Weber MW, Carlin JB, Gatchalian S, Lehmann D, Muhe L, Mulholland EK, et al. Predictors of neonatal sepsis in developing countries. Pediatrics Infect Dis J2003; 22(8):711-7.

8. Shah AJ, Mulla SA, Revdiwala SB. Neonatal sepsis: High antibiotic resistance of the bacterial pathogens in a neonatal intensive care unit of a tertiary care hospital. J Clin Neonatol. 2012; 1:72-5. [PMC free article] [PubMed].

9. Shaw CK, Shaw P, Thapalial A. Neonatal sepsis bacterial isolates and antibiotic susceptibility patterns at a NICU in a tertiary care hospital in western Nepal: a retrospective analysis. KUMJ. 2007; 5:153-60. [PubMed].
10. Bhattacharjee A, Sen MR, Prakash P, Gaur A, Anuprabha S. Increased prevalence of extended spectrum $\beta$ - Lactamase producers in neonatal septicemic cases at tertiary referral hospital. Indian J Med Microbiol. 2008; 26: 356-60. [Pub Med].

11. Buttery JP. Blood cultures in newborn and children: optimizing an everyday test. Arch Dis Child Fetal Neonatal Ed. 2002;87: F25-7

12. Rajaratnam JK, Marcus JR, Flaxman AD, Wang H, Levin-Rector A, Dwyer L, et al. Neonatal, postneonatal, childhood, and under-5 mortality for 187 countries, 1970-2010: a systematic analysis of progress towards Millennium Development Goal 4. Lancet. 2010; 375:1988-2008

13. Sharma CM, Agrawal RP, Sharan H, Kumar B, Sharma D, Bhatia SS. Neonatal Sepsis": Bacteria \& amp; their Susceptibility Pattern towards Antibiotics in Neonatal Intensive Care Unit. J Clin Diagn Res. 2013 Nov;7 (11):2511-3.

14. Vesikari T, Janas M, Gronroos P, 'Tuppurainen N, Renlund M, Kero P, et al. Neonatal septicemia. Arch Dis Child 1985; 60: 542-546.

15. Thakur S, Thakur K, Sood A, Chaudhary S. Bacteriological profile and antibiotic sensitivity pattern of neonatal septicaemia in a rural tertiary care hospital in North India. Indian J Med Microbiol 2016; 34:67-71.

16. Bhat Y R, Lewis LE, Ke V. Bacterial isolates of early-onset neonatal sepsis and their antibiotic susceptibility pattern between 1998 and 2004: An audit from a center in India. Italian J Pediatr 2011; 37: 32 doi: 10.1186/1824-7288-37-32.

17. Van den Hoogen A, Gerards LJ, VerboonMaciolek MA, Fleer A, Krediet TG. Long-term trends in the epidemiology of neonatal sepsis and antibiotic susceptibility of causative agents. Neonatology 2010; 97:22-8.

18. Agnihotri N, Kaistha N, Gupta V. Antimicrobial susceptibility of isolates from neonatal septicemia. Jpn J Infect Dis 2004; 57:273-5.

19. Gerdes JS. Diagnosis and management of bacterial infections in the neonate. Paeditr Clin North Am 2004 Aug; 51(4): 939-59. 
20. Sriram R. Correlation of blood culture results with the sepsis score and the sepsis screen in the diagnosis of neonatal septicemia. Int J Biol Med Res. 2011; 2(1): 360-68.

21. Swarnakar K, Swarnakar M. A study of early onset neonatal sepsis with special reference to sepsis screening parameters in a tertiary care centre of rural India. The Internet J Inf Dis. $2012 ; 10(1)$.

22. Jadhav S, Misra R, Vyawahare C, Angadi K, Gandham N, Ghosh P. Role of sepsis screen in the diagnosis of neonatal sepsis. Medical Journal of Dr DY Patil University. 2013;6(3):254-57.

23. Vinay BS, Girish G N, Adhikari S, Hugara S. Evaluation of septic screen as a diagnostic tool for neonatal sepsis in a tertiary hospital at Mysore. Sch J App Med Sci. 2015;3(2G):1005-10.

24. Bhale CP, Kale AV, Kale SS, Mahajan S, Mulay SS. Utility of Sepsis Screen in the Early Diagnosis of Neonatal Sepsis.IndianJournal of Neonatal MedicineandResearch.2016July,Vol-4(3):IO01-IO07

\section{How to cite this article?}

Juneja S, Chaudhary P, S Thora, Arya S. A study of Neonatal Sepsis and to assess the validity of sepsis screen as a diagnostic tool.Int J Pediatr Res. 2018;5(3):135-141. doi:10.17511/ijpr.2018.i03.06. 